\title{
NEUROLOGICAL MANIFESTATIONS IN HAEMANGIOMA OF THE VERTEBRAE
}

\author{
BY \\ H. ASKENASY and A. BEHMOARAM \\ From the Neurosurgical Department, Beilinson Hospital, Petah Tikva, Israel
}

Haemangioma of the vertebrae is believed to be extremely rare and of little clinical importance, because most often it is detected as an incidental radiological finding in patients examined for complaints thought to be related to other diseases. However, haemangiomata of the vertebrae have been found on examination in large necropsy series in a much larger proportion than suggested by clinical experience. Töpfer (1928), in Schmorl's Institute, reported an incidence of haemangioma of $11.9 \%$ in a study of 2,154 spinal columns and later Junghanns (1932) found this lesion in $10 \%$ of 10,000 necropsies.

Since Perman (1926) emphasized the radiological features characteristic of haemangioma of the vertebrae, there has been increasing interest in this lesion, particularly when it was realized that it may be responsible for a wide variety of symptoms and signs ranging from tenderness and mild pain over the diseased vertebrae to complete transverse myelopathy. Furthermore, neurological disturbances occur more frequently in association with haemangioma of the vertebrae than appears from the literature, and since we started performing systematic radiological examinations in patients referred to our out-patient clinic for back pain we found haemangioma of the vertebrae in a comparatively short time in another three patients who were not admitted to hospital.

The indications for surgical treatment of patients with signs of cord compression are still a matter of dispute. Opinion has been divided ever since Nattrass and Ramage (1932) and Ferber and Lampe (1942) insisted that radiotherapy only should be used in all cases of vertebral haemangioma. We therefore present eight cases of haemangioma of the vertebrae seen during the last few years in the Department of Neurosurgery of the Beilinson Hospital.

The salient features of these patients are summarized in Table I. Our patients experienced various back, shoulder, arm, chest, abdominal, and sciatic pains. Some were thought to have cardiac, joint, or abdominal disease. Others exhibited neurological disturbances.

\section{The Two Clinical Groups}

In accordance with the manifestations, patients with symptomatic haemangioma of the vertebrae are classified into two clinical groups: (1) Patients with symptoms only; (2) patients showing signs of root and/or spinal cord compression in addition to their complaints.

Group 1.-Tenderness or pain are the leading symptoms of this group, in most cases probably directly attributable to irritation by the lesion or the innervation of the bone itself; however, they may also be early manifestations of root involvement.

Tenderness over the diseased vertebra may be spontaneous or elicited on palpation, percussion, or movement. This complaint is far from being characteristic of the lesion but whenever encountered radiographs of the region should be taken.

Pain may be spontaneous or elicited; it may be localized or diffuse, continuous, or, more often, remittent; it may be mild or very severe. Sometimes the pain has a radicular distribution.

The symptoms depend on the level of the bone lesion. Patients with cervico-dorsal lesions may complain of stiff neck, suboccipital pain, pain in the arms and in the praecordial region. Most often, however, a manifesting haemangioma of the vertebrae is found in the dorsal spine and then the common symptoms are of intercostal neuralgia, abdominal or back pain. Low back and sciatic pain, sacralgia, and pain radiating to the inguinal region are seen in thoraco-lumbar and lumbar lesions. It is significant that in quite a number of patients, whose complaints had been labelled for a long time as nephrolithiasis, gastric or duodenal ulcer, pancreatitis and cholelithiasis, haemangioma of the dorsolumbar vertebrae was found on radiological examination. Improvement or recovery 
TABLE I

CLASSIFICATION OF EIGHT PATIENTS WITH HAEMANGIOMA OF THE VERTEBRAE WITH NEUROLOGICAL MANIFESTATIONS

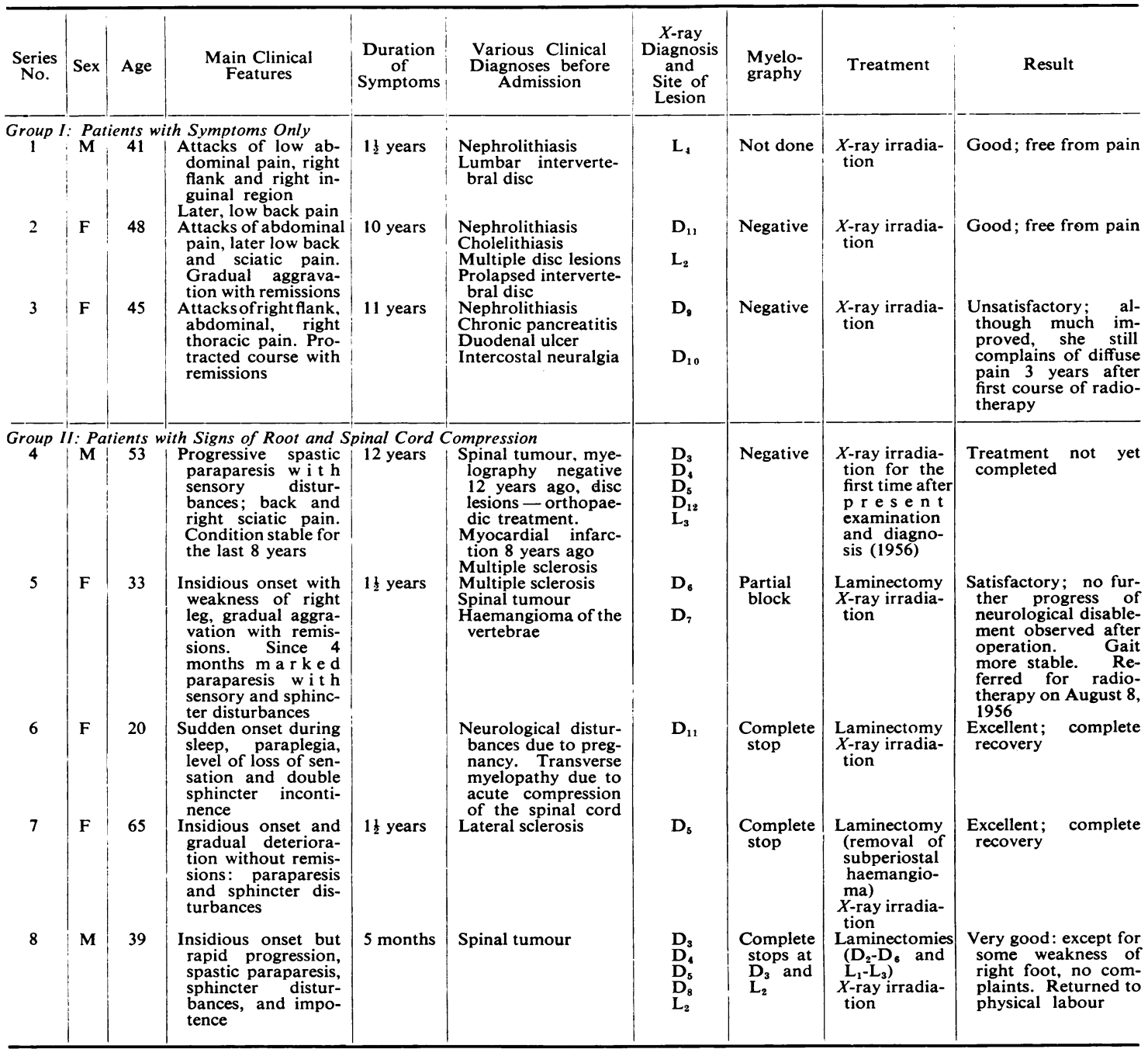

from the pain after irradation of the bone lesion provides, in these patients, convincing proof that haemangioma of the vertebrae was the offender.

Case 1.-A 48-year-old woman complained for 10 years of severe epigastric pain radiating to the inguinal regions, together with nausea and vomiting at their peak. At the onset complaints were localized on the right side only. She had been in hospital and treated for nephrolithiasis for two months. However, no radiological or laboratory evidence of renal or ureteral calculi was found. After a remission of one and a half years she again developed severe epigastric pain radiating now to both inguinal regions. On admission to hospital again, although the cholangiogram was negative, a tentative diagnosis of cholelithiasis was made and the patient was treated accordingly. She was reported much improved on discharge from hospital. Until five years before admission to our department she was not severely ill, but nonetheless she suffered occasionally mild to moderate discomfort localized in the region of the xyphoid process and the epigastrium. Five years before admission she again had a severe attack of abdominal pain radiating to the back.

Three years ago, on re-examination for similar complaints, spondylo-arthritic degeneration of the dorsolumbar spine and mild scoliosis were found. Orthopaedic treatment was instituted. Since then she had symptoms only after effort. One month before admission the patient was suddenly taken with very severe abdominal 
and low back pain, referred along the right sciatic nerve. She was admitted to the Department of Neurosurgery on January 2, 1955, with the provisional diagnosis of multiple disc lesions with, possibly, a prolapse in the lumbar region.

On admission, the physical examination showed mild tenderness on palpation over the low dorsal and upper lumbar spine. The overlying skin appeared normal. The neurological examination was entirely negative. The cardiovascular system, the lungs, and abdomen showed no abnormalities. The blood pressure was $140 / 95 \mathrm{~mm}$. Hg. Routine laboratory investigations were normal. The blood sedimentation rate was $7 / 14 \mathrm{~mm}$. in one hour (Westergren). Radiographs of the dorso-lumbar spine disclosed slight scoliosis and spondylo-arthritis. The eleventh dorsal and the second lumbar vertebral bodies had a reticular appearance with the paralleling and hypertrophy of vertical trabeculae pathognomonic for haemangioma.

On lumbar puncture, the cerebrospinal fluid was clear and normal in every respect. The jugular compression test was negative. The patient was discharged on January 10 and referred for $x$-ray irradiation centred on the diseased vertebrae. She was seen in the out-patient clinic after the completion of radiotherapy. Until examined, for the last time, one year later she had been entirely free of complaints.

This case illustrates symptomatic haemangioma of the spine with subjective complaints only. Nephrolithiasis, cholelithiasis, and, finally, spondyloarthritis with disc prolapse were successively diagnosed over a period of 10 years. The course of the illness was characterized by remissions but with a general trend towards aggravation. There was noticeable improvement after radiotherapy and the pain disappeared.

Group 2.-The second group comprises patients exhibiting symptoms and signs of root or spinal cord compression or of both. At the onset there may be only tenderness or pain as in the first group, neurological signs developing later with the growth of the lesion. However, in most patients of this group, the onset of motor weakness of the legs with paraesthesiae is insidious, and, later on, ascending neurological disturbances develop. The latter may be gradual and slow or dramatic. Sphincter disturbances and impotence are usually seen in more advanced stages of cord compression but they may appear early. Predominant involvement of the lateral and, less frequently, of the dorsal columns of the spinal cord is characteristic. Such a distribution of cord lesions does not depend solely on direct compression but is probably due to stress forces exerted on the lateral columns of the cord resulting from stretching of the anchoring dentate ligaments by the backward pressure on the cord (Kahn, 1947). In some cases complete transverse myelopathy develops with paraplegia and double sphincter incontinence.

Case 2.-A 20-year-old woman was admitted to the Neurosurgical Department of the Beilinson Hospital on August 3, 1951, with the diagnosis of tumour of the spinal cord.

She had been entirely healthy until July 20,1951 , when she awoke during the night with lancinating pain and discovered that both her legs were paralysed. Four days later (July 24, 1951) she was delivered of a normal child. When admitted to the medical department of another hospital (July 29, 1951), flaccid paraplegia, double sphincter incontinence, and anaesthesia from the D12 dermatome downwards were found. A lumbar puncture was done and complete blockage of the subarachnoid space was found on the jugular compression test. The Pandy reaction was positive, cells $10 / 3$, protein $150 \mathrm{mg} . \%$. Radiographs of the spine had not been taken.

On admission to our department (August 3, 1951), the examination showed neurological signs of transverse myelopathy at the level D12 as detailed above. The general examination was negative. The blood pressure

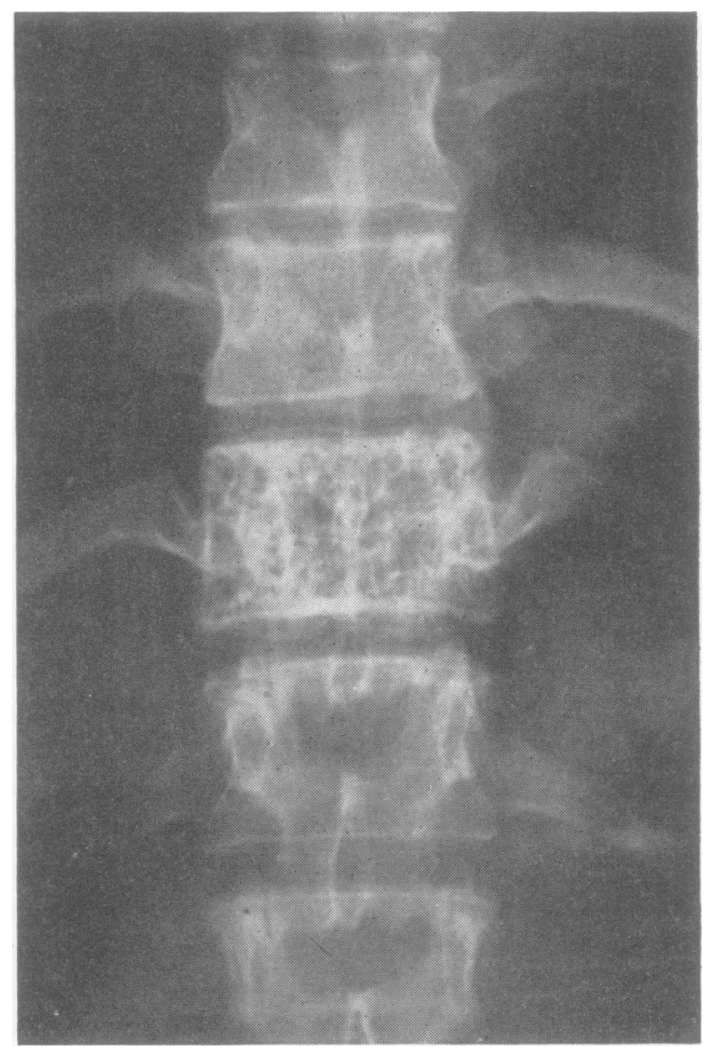

FIG. 1.-Antero-posterior view of the dorsal spine, showing the net-like reticular aspect, with hypertrophy of vertical trabeculae of $D_{11}$, characteristic of haemangioma of the vertebrae. The concave borders of the vertebral body are lost; the pedicles are hardly recognizable. 


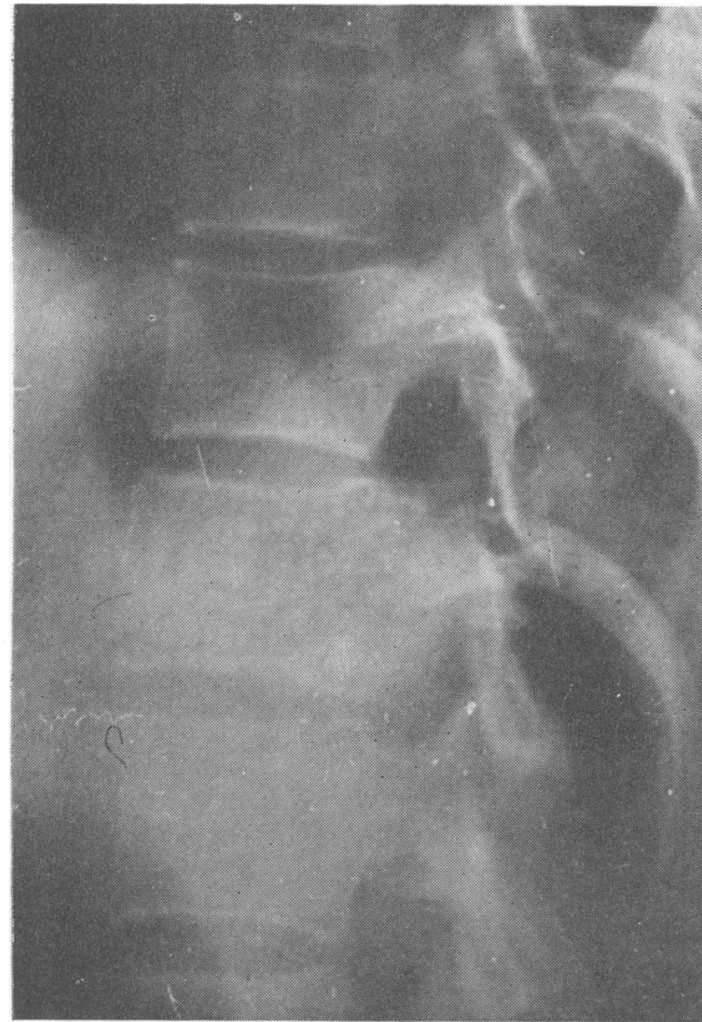

FIG. 2.-Lateral view of the spine, showing vertical trabeculation of the body, its posterior border bulging into the spinal canal; the pedicles and arches are involved in the lesion.

was $100 / 70 \mathrm{~mm}$. $\mathrm{Hg}$. The blood sedimentation rate was $24 / 75 \mathrm{~mm}$. in one hour. Other routine laboratory examinations were normal.

$X$-ray films of the dorsal spine showed a reticular appearance with gross trabeculation and expansion of the contours of the eleventh dorsal vertebra, typical of haemangioma (Fig. 1). The pedicles and the laminae were involved by the lesion as seen on lateral views (Fig. 2). There was a complete stop at the level of the bone lesion on myelography (August 4, 1951).

At operation on the same day (laminectomy D10-D12), there was profuse bleeding on removing the spinous processes and the arches. Bleeding was controlled with beeswax. On completion of the laminectomy, the cord, which was slightly humped over the bulging posterior surface of the vertebra, recovered normal pulsation. Histological examination of the removed bone showed a haemangioma of the vertebra (Prof. J. Casper).

The post-operative course was uneventful. The patient was ambulatory on the thirteenth day after operation (August 18, 1951); however, she needed support in daily walking practice. She was referred for radiotherapy on September 9, 1951.

The patient came periodically to the out-patient clinic; good progress was noted and when seen for the last time in 1953 she was leading a normal life and the neurological examination was negative.

In this case of vertebral haemangioma in a pregnant woman, signs of transverse myelopathy developed suddenly four days before delivery.

Massive neurological disturbances of sudden onset are certainly an unusual development in haemangioma of the vertebrae. Collapse of the vertebral body due to destruction by haemangioma is rare (Bell, 1955).

Neurological signs progressed slowly in reported cases with collapse of the vertebral body due to haemangioma: over a period of 16 years in Muthman's case (quoted by Bell, 1955), eight years in Sandahl's (quoted by Bell, 1955), one year in that reported by Globus and Doshay (1929), and more than six months in Bell's (1955) patient. But if the rich vascularity of the lesion is borne in mind the operative mechanisms of acute cord compression in haemangioma of the vertebrae becomes intelligible. Vascular lesions, and in particular those located within the spinal canal or protruding into it, are bound to produce severe disturbances when they suddenly increase in size. Moreover, it is known that signs became aggravated during the night in intraspinal space-occupying lesions due to physiological venous stasis and congestion of the epidural spinal venous plexus during sleep. Congestion is further enhanced by increased blood volume and raised intraabdominal pressure in advanced pregnancy, and these factors probably favoured the sudden onset of acute cord compression during sleep in Case 2.

Case 3.-A 65-year-old woman was admitted to the Department of Neurosurgery on September 22, 1952, for operation on a suspected tumour of the mid-thoracic spinal cord.

Her complaints had started one and a half years before admission with weakness of the right leg. She tired easily and needed support for walking. Six months before she experienced numbness and pricking of the right sole, later involving the right foot and then the other leg in the same sequence. A month later she noticed that she did not feel her stockings, she "walked on wool". Ascending disturbances of sensation developed gradually with advancing paraparesis. A week before admission she complained of severe back pain and difficulty in urination.

Neurological examination on admission showed spastic paraparesis, more pronounced on the right side with hyperactive tendon jerks, right ankle clonus, and bilateral Babinski signs. Hypoaesthesia for light touch, cold and heat, hypalgesia, and loss of position sense were found from the D6 dermatome increasing downwards. Anaesthesia and analgesia of the soles and lateral sides of both feet were noted and there was hyperalgesia and hyperaesthesia in a zone corresponding to D5-D6. Physical and routine laboratory examinations were 


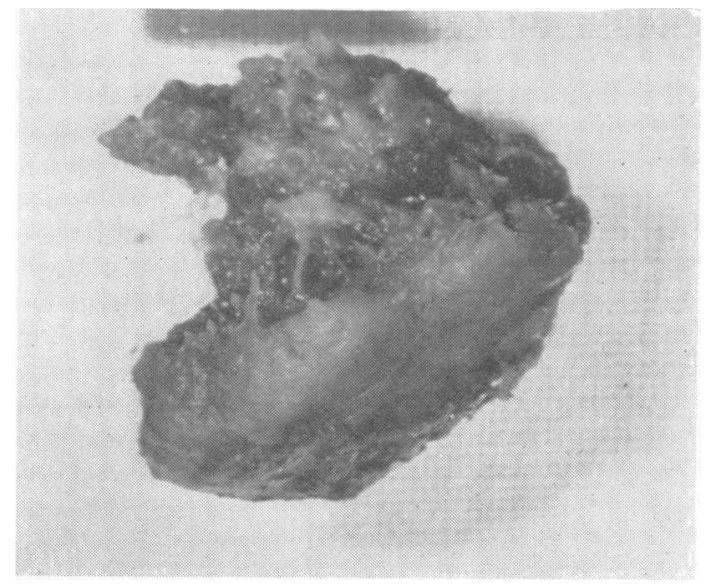

FIG. 3.-Surgical specimen of subperiostal haemangioma measuring $2-2 \frac{1}{2} \mathrm{~cm}$.

negative. The blood pressure was $160 / 100 \mathrm{~mm}$. $\mathrm{Hg}$. The blood sedimentation rate was $10 / 16 \mathrm{~mm}$. (Westergren). On lumbar puncture, clear cerebrospinal fluid under normal tension was obtained. The Pandy reaction was negative, cells $4 / 3$, protein $28 \mathrm{mg}$. and glucose $65 \mathrm{mg}$. per $100 \mathrm{ml}$. The Mastix and Kahn tests were negative. The jugular compression test showed a slow elevation and a retarded drop of the cerebrospinal fluid column suggestive of incomplete obstruction of the subarachnoid space.

Straight radiographs of the spine disclosed slight trabeculation of the fifth vertebral body; its contour, however, was normal. The pedicles and the laminae appeared normal. A tentative diagnosis of haemangioma of the vertebrae was proposed but the possibility of a coexistent intraspinal tumour was entertained. Myelography (October 2, 1952) showed a complete stop at D5. At operation on the same day (laminectomy D4-D6), the exposed spinal cord bulged over a dark, red-bluish epidural mass protruding from the posterior surface of the fifth dorsal vertebrae. The tumour was well delineated, friable, and bled profusely when incised. After careful lateral retraction of the cord the tumour was easily removed. There was severe bleeding which was controlled with bone wax. The mass measured 2 to $2 \frac{1}{2} \mathrm{~cm}$. (Fig. 3). Histologically (Prof. H. Casper) it showed the structure of a haemangioma.

The post-operative course was uneventful. The patient was discharged from hospital on October 17, 1952, and referred for radiotherapy. She was seen for the last time in the out-patient clinic in 1954 when the neurological examination was negative, except for slight hypoaesthesia of the right foot.

The gradual progression of signs, without remission, in this case was suggestive of a spinal tumour. On operation an intraspinal subperiostal haemangioma was found. A subperiostal haemangioma of the vertebrae may attain relatively large proportions before causing radiologically recognizable alteration of the vertebral structure. In such cases intraspinal tumour is diagnosed and after positive myelography surgery is invariably undertaken. Globus and Doshay (1929) and later Karshner, Rand, and Reeves (1939), who added another case to the previously reported 10 such cases treated surgically, emphasized the most promising results from operation on haemangioma of the vertebrae in this location.

Case 4.-A 39-year-old male manual worker was admitted to the Department of Neurosurgery on January 13, 1955, with the diagnosis of spinal tumour.

He had been entirely well until five months before admission to hospital, when he experienced numbness of the right foot. He massaged his leg because it "felt frozen". A few days later dysaesthesiae and weakness developed in both legs; these complaints became gradually worse and after a week he began to suffer back pain and right sciatic pain. Walking became unsteady and he could not climb stairs. Two months before admission he developed sphincter disturbances with difficulty on urination and he became impotent.

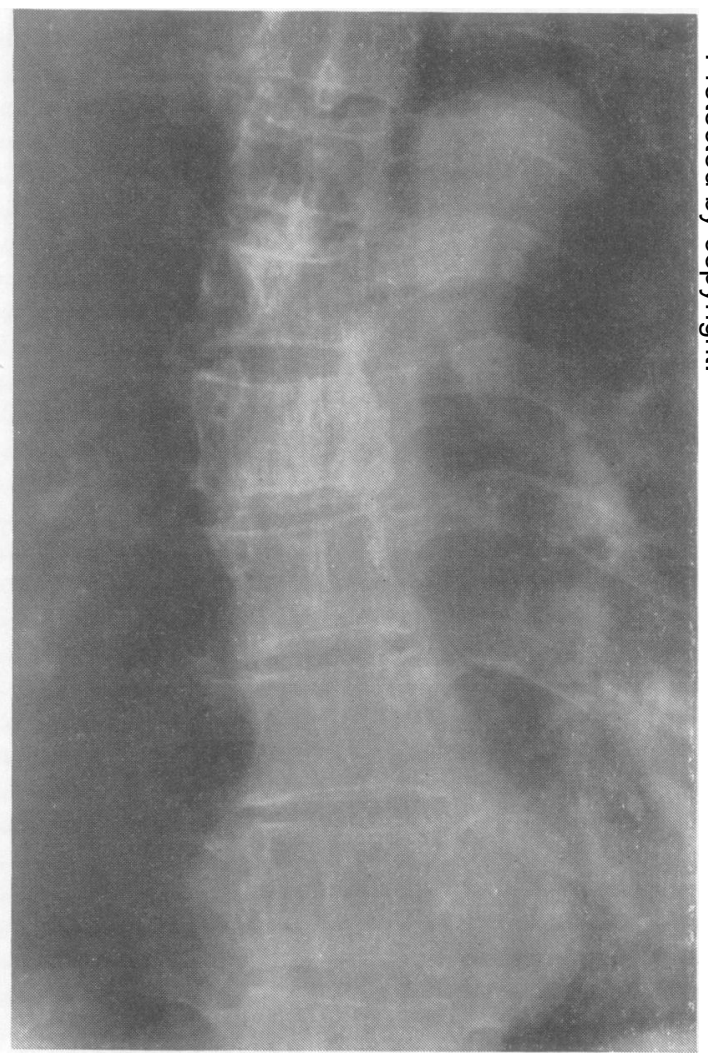

FIG. 4.-Antero-posterior view of the spine. Vertical trabeculation and "expanding" of the $D_{3}, D_{4}, D_{5}$, and $D_{8}$ vertebral bodies is visible. Note the " spindle-shaped" dense shadow around $D_{8}$ radiologically suggesting a cold abscess. 
The physical examination showed spastic paraparesis with hyperactive tendon reflexes, except for an absent right patellar reflex; bilateral Babinski's sign and bilateral ankle clonus were noted but the abdominal reflexes were not diminished and they were equal. Superficial sensation for light touch, cold and heat, and pin prick was diminished from D7 downwards. There was almost complete loss of superficial and deep sensation corresponding to L2, L3, and L4, and associated muscle weakness and noticeable wasting of the muscles of the thigh. The patient's gait was spastic and ataxic. The general examination did not reveal any abnormality. The blood pressure was $140 / 70 \mathrm{~mm}$. Hg. Routine laboratory examinations gave normal results. The cerebrospinal fluid was slightly xanthochromic, and tension was low. The Pandy test was positive, cells 28/3 (lymphocytes), protein $110 \mathrm{mg}$. per $100 \mathrm{ml}$.; Mastix and Kahn tests were negative. Straight $x$-ray films showed scoliosis of the dorso-lumbar spine with gross modifications of D3, D4, D5, D8, and L2 vertebrae, typical for haemangioma. In addition a spindle-shaped dense shadow around the eighth dorsal vertebra was seen. This finding seemed suggestive of a cold abscess (Fig. 4). However, the intervertebral discs appeared intact and there were no historical, clinical, or radiological data supporting the diagnosis of tuberculosis. A complete stop at the level of the second lumbar vertebra was demonstrated on lumbar myelography. A lesion at that level could account for the radicular syndrome of the right leg, and possibly for the sphincter and sexual disturbances, but it did not explain the complete neurological picture, so myelography by the cisternal route was supplemented. This showed another stop, in the standing position, at D4.

Decompressive laminectomy was performed at both levels cn the same day: first D2-D6 and then L1-L3. There was severe bleeding and haemostasis took time. The spinal canal was inspected after complete control of the bleeding. The cord, which was paler than normal and slightly humped at the upper operation site, recovered normal pulsation.

The post-operative course was uneventful. The neurological disturbances gradually became less severe. The level of sensory impairment receded to D9. From the eleventh day after operation the patient walked with support and did not complain about pain. The control myelographic examination performed in view of the lesion at D8, showed freely flowing contrast medium along the length of the spinal canal. The patient was discharged 16 days after operation for $x$-ray irradiation centred on both operative fields and on the eighth dorsal vertebra. $\mathrm{He}$ was periodically re-examined in the out-patient clinic. One year after operation his gait was steady without support, the reflexes were still active, but he was free from pain and had resumed his former occupation.

In this case of multiple haemangioma of the spinal column (D3, D4, D5, D8, L2), there developed progressive neurological manifestations of rather short duration. Bailey and Bucy (1929) and Schlezinger and Ungar (1939) pointed out that involvement of the pedicles and the arches by the lesion is responsible for the greater incidence and earlier onset of neurological disturbances. Although in the present case the arches and the pedicles of the vertebrae were noticeably involved, neurological disturbances seem to have developed in a rather advanced stage of the process. In view of the extensive osseous lesions the manifestations in this case were unimpressive when compared to those in Case 7, and in particular, with the dramatic onset and massive neurological picture in Case 6 (Table I).

The radiological finding of a spindle-shaped shadow around the eighth dorsal vertebra is of interest. The haemangioma had obviously extended to the paravertebral soft tissues, thus giving the radiological picture of a cold abscess. Brøbeck (1950) reported that a similar finding was encountered in Sheid and Buckhardt's case. However, as pointed out, the lack of involvement of intervertebral joints should help to differentiate the lesion from suspected tuberculosis of the spine.

\section{Discussion}

Among the haemangiomata, those arising in proximity to the central nervous system, that is, within the cranial cavity or in the spinal canal, are of special importance. It is easy to understand that severe disturbances may result from an even relatively slight constriction of the lumen of the spinal canal. Haemangiomata of the skin, the liver, and the spleen, for instance, may attain enormous proportions without causing serious functional damage, while a small haemangioma, either by compressing the spinal cord or through secondary vascular disturbances, may bring about serious neurological disability.

Haemangiomata of the vertebrae are benign, expansive, dark red vascular growths. They are made up of cavities which may form cavernous bodies when sufficiently large. The cavities are lined with endothelial cells. The haemangioma does not metastatize, but with growth it displaces the normal bone marrow, which partially degenerates into fatty tissue. Most often it involves the vertebral body, but it may also spread to the pedicles and the arch (Cases 4, 6, 8 in Table I). The lesion may develop initially in the arch, the pedicle, and the spinous process; it may infiltrate the paravertebral soft tissues (Case 8); it may finally erupt into the spinal canal developing subperiostally (Case 7). Brøbeck (1950) cites the case of Jacobovici in which the angioma spread into the soft tissues of the back in the form of a prominent pulsating subcutanous growth. In the course of its development the haemangioma erodes the bony trabeculae of the spongiosa. Additional changes in the cancellous 
bone are produced by ischaemia due to stasis and thrombosis in the large channels and cavities of the vascular lesion. Local ischaemia is known to cause osteolytic and secondary reparatory, osteoblastic activity in the bone.

The structure and pattern of bone depend in part on its vascularization. As long ago as 1905 Gerbhardt considered that the architecture of bone is largely determined by its vascular system. Weidenreich (1923), quoted by Trueta (1956), noticed that the lamellae of cancellous bone are always arranged around spaces containing vessels and follow their direction. Recently Trueta (1956), in research on osteoarthritis, has found suggestive evidence that realignment of the trabecular pattern is also determined by the ability of the spongiosa to orientate its trabeculae along the lines of forces of tension and pressure. It seems, therefore, that the force of gravitation acting in man, in the erect position, is responsible for buttressing the vertical trabeculae in haemangioma of the vertebrae, which in their turn are responsible for the pathognomonic "vertical trabeculation" seen on radiological examination. It is understandable, moreover, that the loadsupporting dorsal and, particularly, lumbar, vertebrae undergo more readily these specific modifications. On the other hand, in haemangioma of the cervical vertebrae, where the call for vertical buttressing is less, as well as in the pedicles, the arches, and the spinous processes of the vertebrae, whose main function is not to bear weight, the pattern of rearrangement is manifested on the $x$-ray plate as the "soap-bubble effect" or the "sun-burst" trabeculation, radiating from a common centre.

The tendency of haemangioma to occur in the vertebrae more often than in other sites of the body is believed to be related to the extensive venous plexus in the mesenchymal tissue of the embryo, which later form the vertebral column. Hanson (1926) pointed out that the frequent occurrence of congenital haemangiomatous new growths may be expected to result from the extremely rich venous plexuses of the embryo. Its remnants in the adult vertebra have been demonstrated in the form of canals traversing the vertebral body, visible on radiography during the first two decades of life. Similarly, the rich venous network of the diploe is considered a reasonable explanation for the not infrequent occurrence of haemangioma of the skull (Schlezinger and Ungar, 1939).

The majority of the growths were situated in the dorsal column (Table I). The site of predilection of the lesion in cases complicated by neurological disturbances was mid- and lower dorsal and in the higher lumbar regions in this series, whereas Globus and Doshay (1929), Schlezinger and Ungar (1939), and Ghormley and Adson (1944) pointed to the mid-dorsal (D3-D5) location of haemangioma as more likely to produce neurological manifestations. Lumbar haemangioma of the vertebra is more often seen in asymptomatic cases. Involvement of the cervical column seems to be rare. Junghanns (1932) reported an incidence in women of haemangioma of the spine of $12.5 \%$ as compared with $8.9 \%$ in men. The age incidence on diagnosis ranges from puberty to a fairly advanced age, as, for example, 65 years in Case 7. Brøbeck (1950) cites Zdansky for having reported the oldest patient, 64 years of age. Although the overall incidence of haemangioma of the vertebrae was found to be below $5 \%$ in persons less than 30 years old, approximately $50 \%$ of the cases, which were accompanied by neurological disturbances referable to compression myelopathy, occurred in the lower age group (Schlezinger and Ungar, 1939). In our material the youngest patient was aged 20 (Case 6). The average age incidence of 43 years in the present series places them in the older age group, in disagreement with the accepted belief that severe neurological complications occur mostly in younger patients.

Of great diagnostic value is the radiologica examination of the clinically suspected region of the spine. However, $x$-ray examination of the entire skeletal system is highly recommended in such cases? Perman (1926), Bailey and Bucy (1929), and Bucy and Capp (1930) described the radiological features of haemangiomata of the vertebrae, characterize by reduction of bone density between densee hypertrophied vertical trabeculae. Perman (1926) maintained that the radiological picture is pathognomonic of the disease. However, infrequently the radiological findings may raise diagnostic difficulties. In Case 8, for example, the spindle-shaped delineated shadow, due to the spread of the lesion in the paravertebral tissues, was similar to the picture seen in Pott's disease and suggested the presence of a paravertebral cold abscess. Osseous lesions encountered in malignant tumour metastases, giant cell tumour, osseous lymphogranulomatosis, myelomatosis, Kümmel's and von Recklinghausen's diseases may lead to diagnostic difficulties.

It should be pointed out that two cases from this hospital of haemangioma of the vertebra with symptoms only were treated erroneously for perforated duodenal ulcer and for chronic pancreatitis. In both patients haemangioma of the vertebrae was later found incidentally on abdominal radiological examination.

The neurological manifestations due to compression myelopathy caused by haemangioma of the vertebrae are comparable, generally with those produced by any other type of extradural tumour, 
although Ghormley and Adson (1944) maintained that they "probably resemble more the symptoms produced by a metastatic lesion of the spinal cord ". Bilateral neurological signs were reported as a regular feature resulting from the almost symmetrical involvement of the cord by the lesion (Schlezinger and Ungar, 1939). This is not verified in our series. The neurological manifestations in the early stage of the disease were invariably unilateral except in the patient with a sudden onset of paraplegia (Case 6).

A clinical course with remission of neurological manifestations is characteristic for vascular lesions. Alpers and Pancoast (1932) report a case of haemangioma with varying symptomatology in which subarachnoidal block, demonstrated at first by Queckenstedt's test, could not be confirmed on a second examination. This would suggest the possibility that there may be a certain amount of expansion of the haemangioma due to filling of the blood spaces. Such an explanation seems acceptable in the case of a subperiostal growth of the lesion. However, considering the rigid bony structure confining the lesion when it is within the vertebral body, variations in symptoms during the course of the disease are more likely to be related to venous stasis and congestion of the epidural venous plexus, and subsequent disturbance in cerebrospinal fluid circulation.

The problem of the correct treatment of patients exhibiting neurological disturbances due to haemangioma of the vertebrae is still unsettled. Bailey and Bucy in 1929 clearly stated that the only treatment of any avail in this disease when there are signs of compression of the spinal cord is laminectomy. They advised radiation of the lesion only after decompression of the cord. They pointed out that the operation is difficult and may be fatal on account of uncontrollable bleeding.

In view of the danger involved in surgery and the reported high mortality rate $(20-25 \%)$, Nattrass and Ramage (1932), Ferber and Lampe (1942), Lindqvist (1951), and others have employed deep $x$-ray therapy as the "primary and sole method of treatment ". Although the value of radiotherapy has been conclusively demonstrated in haemangioma of the vertebrae, one cannot easily be reconciled with such an attitude when the cord is compressed. The use of radiotherapy alone in such cases seems not only inadequate, as control radiographs show little or no demonstrable improvement in the bony lesion, but it is hazardous to the patient because the cord may be irreparably damaged before any beneficial effect from irradiation can be anticipated.

In cases where the haemangioma has not spread to the arches and the spinous process the operative procedure itself does not carry undue risks. The possibility of such a spread should be judiciously evaluated before operation. Experience has shown that with modern anti-shock and blood replacement therapy, the surgery of haemangioma of the vertebrae is relatively safe. Decompressive laminectomy was carried out by us five times in four patients, one of whom (Case 8) was operated on at two sites, dorsal and lumbar, at the same session without ill effect. There was no mortality and none of the patients were shocked. The post-operative course was uneventful and the patients were ambulatory in the third week after operation. The end-results were good (Table I).

In conclusion we favour the following attitude towards patients with symptomatic haemangioma of the vertebrae:

(1) Patients with only subjective complaints should be irradiated.

(2) Patients exhibiting neurological signs only of root compression should be irradiated.

(3) Patients exhibiting progressive neurological signs of cord compression should undergo myelography. In the presence of a block of the subarachnoid space, immediate decompressive laminectomy should be carried out, supplemented by radiotherapy.

\section{Summary}

Eight cases of haemangioma of the vertebrae with neurological manifestations were observed in a period of six years. Four of these are presented in detail. The cases are classified into two main clinical groups: those with symptoms only and those with objective neurological signs.

In the first group, root involvement is held responsible for a variety of complaints which often simulated acute or chronic medical and surgical conditions. In these patients haemangioma of the vertebrae was incidentally discovered on radiological examination. Improvement after radiotherapy supported the diagnosis of symptomatic haemangioma of the vertebrae.

The second group of patients exhibited objective neurological signs of root lesion or of cord compression. Five examples of spinal cord compression due to haemangioma of the vertebrae are given. Four of these were operated on after myelographic confirmation.

The authors believe that the neurological complications are not due solely to compression but are enhanced by congestion, bleeding, thrombosis 
within the lesion in combination with stasis of blood flow in the epidural venous plexus, and secondary disturbances of the cerebrospinal fluid circulation. Aggravation of the clinical manifestations may be favoured by various factors. Endocrine disturbances and elevated intra-abdominal pressure in advanced pregnancy, together with physiological nocturnal venous stasis, are believed to have contributed in one case to the sudden onset of paraplegia.

The authors rally to the opinion that operation is the treatment of choice in cases of haemangioma of the vertebrae with myelographic confirmation of compression of the cord. Decompressive laminectomy should be supplemented by radiotherapy. Surgery in the hands of the authors carried no mortality.

\section{REFERENCES} Alpers, B. J., and Pancoast, H. K. (1932). Surg. Gynec. Obstet.,

Bailey, P., and Bucy, P. C. (1929). J. Amer. med. Ass., 92, 1748.

Bell, R. L. (1955). J. Neurosurg., 12, 570.

Brøbeck, O. (1950). Acta. radiol. (Stockh.), 34, 235.

Bucy, P. C., and Capp, C. S. (1930). Amer. J. Roentgenol., 23, 1.

Ferber, L., and Lampe, J. (1942). Arch. Neurol. Psychiat. (Chicago), $47,19$.

Ghormley, R. K., and Adson, A. W. (1944). J. Bone J. Surg., 23, 887. Globus, J. H., and Doshay, L. J. (1929). Surg. Gynec. Obstet., 48, 345 .

Hanson, R. (1926) Arch. chir, scand 60, 309.

Junghanns, H. (1932). Arch. klin. Chir., 169, 321.

Kahn, E. A. (1947). J. Neurosurg., 4, 191.

Karshner, R. G., Rand, C. W., and Reeves, D. L. (1939). Arch. Surg. (Chicago), 39, 942 .

Lindqvist, I. (1951). Acta radiol. (Stockh.), 35, 400.

Nattrass, F. J., and Ramage, D. (1932). J. Neurol. Psychopath., 12, 231.

Perman, E. (1926). Acta chir. Scand., 61, 91.

Schlezinger, N. S., and Ungar, H. (1939). Amer. J. Roentgenol., 42, 192.

Töpfer, D. (1928). Frankfurt Z. Path., 36, 337. Quoted from

Trueta, J. (1956). Lancet, 1, 585. 